\title{
On solving some classes of nonlinear fractional differentional equations using fractal index method
}

\author{
Sayed K. Elagan ${ }^{1,2}$, Mohamed S. Mohamed ${ }^{1,3}$, Khaled A. Gepreel ${ }^{1,4}$, Rabha W. Ibrahim ${ }^{5}$, \\ Afaf Elesimy ${ }^{1}$ \\ ${ }^{1}$ Mathematics Department, Faculty of Science, Taif University, Taif, Saudi Arabia \\ ${ }^{2}$ Mathematics Department, Faculty of Science, Menofya University, Shebin Elkom, Egypt \\ ${ }^{3}$ Mathematics Department, Faculty of Science, Al-Azhar University, Cairo, Egypt \\ ${ }^{4}$ Mathematics Department, Faculty of Science, Zagazig University, Kuala Lumpur, Egypt \\ ${ }^{5}$ Institute of Mathematical Sciences, University Malaya, 50603, Kuala Lumpur, Malaysia
}

\section{Email address:}

sayed_khali12000@yahoo.com (S. K. Elagan)

\section{To cite this article:}

Sayed K. Elagan, Mohamed S. Mohamed, Khaled A. Gepreel, Rabha W. Ibrahim, Afaf Elesimy. On Solving Some Classes of Nonlinear Fractional Differentional Equations Using Fractal Index Method. Science Journal of Applied Mathematics and Statistics.

Vol. 2, No. 6, 2014, pp. 112-115. doi: 10.11648/j.sjams.20140206.12

\begin{abstract}
We provide a new solution of diffusion fractional differential equation using fractal index and fractional sub-equation method. Also we shall impose a new solution for fraction Birnolli equation of arbitrary order using the fractal index method. As a result many exact solutions are obtained. It is shown that our considered method provides a very effective tool for solving fractional differentional equations.
\end{abstract}

Keywords: Fractional Sub-Equation Method, Fractal Index Method

\section{Introduction}

Fractional differential equations are viewed as alternative models to nonlinear differential equations. Varieties of them play important roles and tools not only in mathematics but also in physics, dynamical systems, control systems and engineering to create the mathematical modeling of many physical phenomena. Furthermore, they employed in social science such as food supplement, climate and economics. Fractional differential equations concerning the Riemann-Liouville fractional operators or Caputo derivative have been recommended by many authors (see [1-5]).

Transform is a significant technique to solve mathematical problems. Many useful transforms for solving various problems were appeared in open literature such as wave transformation, Laplace transform, the Fourier transform, the $\mathrm{B}$ " ucklund transformation, the integral transform, the local fractional integral transforms and the fractional complex transform and Mellin transform (see [6-10]), and the fractional complex transform, which was first proposed by $\mathrm{He}$ and $\mathrm{Li}$ [7-10], among which the fractional complex transform [11] is the simplest approach, it is to convert the fractional differential equations into ordinary differential equations, making the solution procedure extremely simple. Such transformation is valid only for general "wave" solutions for fractional differential equations. However, not every fractional differential equation has a "wave" solution, hence its application is limited, for this reason He and S.K et al [12, 13] suggested a general transform which depend on the fractal index method.

One of the most tools in the theory of fractional calculus is viewed by the RiemannLiouville operators. It imposes advantages of fast convergence, higher stability and higher accuracy to derive different types of numerical algorithms. In this note, we shall deal with scalar linear time-space fractional differential equations. The time and the space are taken in sense of the Riemann-Liouville fractional operators. Also, This type of differential equation arises in many interesting applications [14-27]. Several researchers have studied fractional dynamic equations generalizing the diffusion or wave equations in terms of R-L or Caputo time fractional derivatives, and their fundamental solutions have been represented in terms of the Mittag-Leffler (M-L) functions and their generalizations. In this article, We provide a new solution of diffusion fractional differential equation using a modefied fractional sub-equation method. Also we shall impose a new solution for fraction Birnolli equation of arbitrary order. As a result many exact solutions are obtained. 
It is shown that our considered method provides a very effective tool for solving fractional differentional equations. The fractional operators are taken in sense of the Riemann-Liouville operators [1].

\section{Fractal Index Method}

Consider the following equation

$$
{ }_{t}^{\alpha} D_{t}^{\alpha} u+\left(a+b u+c u^{2}\right) x+e D_{x}^{\beta} u=0, e \neq 0, a=c=0,
$$

where $u(t, x)$ is the unknown function .

Let $X=x^{\alpha}$ and $f=X^{n}, n \neq 0$, then we obtain

$$
\begin{aligned}
\frac{\partial f}{\partial x^{\alpha}} & =\frac{\partial f}{\partial X} \frac{\partial^{\alpha} X}{\partial x^{\alpha}} \\
& =\frac{\Gamma(1+n \alpha)}{\Gamma(1+n \alpha-\alpha)} x n \alpha-\alpha=\frac{\partial f}{\partial X} \theta_{\alpha} \\
& =n \theta_{\alpha^{x}} n \alpha-\alpha
\end{aligned}
$$

See [12], where $\theta_{\alpha}=\frac{\Gamma(1+n \alpha)}{\Gamma(1+n \alpha-\alpha)}$ and called the fractal index. Now we proceed to impose a new solution for the Eq. (1) using the fractal method.

Let the solution takes the form

$$
u(t, x)=t \mu(x)+v(t, x) \cdot\left(v(t, x)=O\left(t^{2}\right)\right) .
$$

We propose to show that (1) has a unique analytic solution by using the Banach fixed point theorem. Calculations imply

$$
{ }_{t} \alpha D_{t}^{\alpha} \alpha=\frac{t}{\Gamma(2-\alpha)}+{ }_{t}{ }^{(\alpha}{ }_{t}^{(\alpha)}
$$

and

$$
e D_{x}^{\beta} u=e t D_{x}^{\beta} \mu(x)+e v_{x}^{(\beta)}
$$

Therefore, $\mu(x)$ satisfies,

$$
\frac{\mu(x)}{\Gamma(2-\alpha)}+b x \mu(x)+e D_{x}^{\beta} \mu(x)=0
$$

which equivalences to

$$
D_{x}^{\beta} \mu(x)=g(x, \mu(x))
$$

where

$$
g(x, \mu(x))=\rho(x) \mu(x),|x|<1,|\rho(x)|<1
$$

And

$$
\rho(x)=-\left(\frac{1}{2 \Gamma(2-\alpha)}+\frac{b x}{e}\right) .
$$

Now $g(x, \mu(x))$ is a contraction mapping; therefore, in view of the Banach fixed point theorem, Eq.(2) has a unique analytic solution in $[-1,1]$ and consequently the problem (2).
To calculate the fractal index for the equation

$$
D_{x}^{\beta} \mu(x)+\rho(x) \mu(x)=0, \mu(0)=1,|\rho(x)|<1,
$$

we assume the transform $X=x^{\beta}$ and the solution can be expressed in a series in the form $\mu(X)=\sum_{m=0}^{\infty} \mu_{m} X^{m}$ where $\mu_{m}$ are constants. Substituting yields

$$
\frac{\partial}{\partial X} \sum_{m=0}^{\infty} \theta_{\beta m} \mu_{m} X^{m}+\rho \sum_{m=0}^{\infty} \mu_{m} X^{m}=0,
$$

where

$$
\rho=\max _{x \in[0,1]} \rho(x) .
$$

Note that $0<|\rho|<1$ and the fractal index $\theta_{\beta m}=\frac{\Gamma(1+m \beta)}{m \Gamma(1+m \beta-\beta)}$, then a computation imposes the relation

$$
\frac{\Gamma(1+m \beta)}{m \Gamma(1+m \beta-\beta)} \mu_{m}+\rho \mu_{m-1}=0, \text { with } \mu(0)=1
$$

consequently we obtain

$$
\mu_{m}=\frac{(-\rho)^{m}}{\Gamma(1+m \beta)} .
$$

Thus we have the following solution

$$
\mu(x)=\sum_{m=0}^{\infty} \frac{(-\rho)^{m}}{\Gamma(1+m \beta)} x^{m \beta}=E_{\beta}(-\rho x \beta),
$$

where ${ }^{E} \beta$ is a Mittag-Leffler. The last assertion is the exact solution for the problem (2) and consequently for (1).

Now we will find the exact solution for the fractional Birnolli equation using the fractal index method. Consider the following fractional Birnolli equation

$$
D_{x}^{\alpha} \psi(x)=\mu \psi^{2}(x)-\lambda \psi(x),
$$

where $\lambda, \mu \in R$ and $\lambda, \mu \neq 0$. To calculate the fractal index for the equation (3), we assume the transform $X={ }_{x} \alpha$ and the solution can be expressed in a series of the form

$$
\psi(X)=\sum_{m=0}^{\infty} \psi_{m} X^{m}, \quad \psi(0)=1
$$

where $\psi_{m}$ are constants. Substitute (4) into (3) and by applying the fractal index we impose

$$
\begin{aligned}
& \frac{\partial}{\partial X} \sum_{m=0}^{\infty} \theta_{\alpha m} \psi_{m} X^{m}=\left(\mu \sum_{m=0}^{\infty} \psi_{m} X^{m}\right)\left(\sum_{m=0}^{\infty} \psi_{m} X^{m}\right)-\left(\lambda \Sigma_{m=0}^{\infty} \psi_{m} X^{m},\right. \\
& \left.\sum_{m=1}^{\infty} \frac{\Gamma(1+m \alpha)}{\Gamma(1+m \alpha-\alpha)} \psi_{m} X^{m-1}=\mu \Sigma_{m=0}^{\infty} \psi_{m} X^{m}\right)\left(\Sigma_{m=0}^{\infty} \psi_{m} X^{m}\right)-\left(\lambda \Sigma_{m=0}^{\infty} \psi_{m} X^{m}\right),
\end{aligned}
$$

where

$$
\theta_{\alpha m}=\frac{\Gamma(1+m \alpha)}{m \Gamma(1+m \alpha-\alpha)}
$$


Comparing the coefficients of $x^{0}$, we have

$$
\Gamma(1+\alpha) \psi_{1}=\mu \psi_{0}^{2}-\lambda \psi_{0}
$$

But $\psi_{0}=1$ so in general we obtain

$$
\psi_{m}=\frac{(\mu-\lambda)^{m}}{\Gamma(1+m \alpha)}, \quad m \geq 1
$$

Hence

$$
\psi(x)=E_{\alpha}((\mu-\lambda) x \alpha)
$$

In the next section, we shall use (5) to locate exact solution of fractional differential equation using fractional Birnolli equation.

\section{The Fractional Subequation Method}

In this section, based on the solution of Birnolli fraction equation, we will impose a technique for solving fractional partial differential equations using the sub-equation method [18]. It will be shown that this technique permits us to get new exact solutions from the known seed solutions.

Our method can be summarized as follows:

Step 1: Using the wave transform

$$
\begin{gathered}
u\left(t, x_{1}, \ldots, x_{j}\right)=u(\eta), \\
\eta=\eta_{0}+\lambda t+\lambda_{1} x_{1}+\ldots+\lambda_{j} x_{j},
\end{gathered}
$$

Where $\lambda, \lambda_{i}(i=1, \ldots, j)$ are constants. Hence the equation

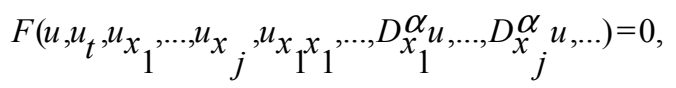

becomes

$$
\Phi\left(u(\eta), u^{\prime}(\eta), u^{\prime \prime} \eta_{u}\right)=0
$$

where ()$=\frac{d}{d \eta}$.

Step 2: Assuming a solution of the form

$$
u(\eta)=\sum_{i=-m}^{-1} a_{i} \psi^{i}(\eta)+a_{0}+\sum_{i=1}^{m} a_{i} \psi^{i}(\eta)
$$

where $a_{i}(i=-m,-m+1, \ldots, m-1, m)$ are constants to be calculated and $\psi$ statisfies the fraction Birnolli equation

$$
D_{x}^{\alpha} \psi(x)=\mu \psi^{2}(x)-\lambda \psi(x),
$$

where $\lambda, \mu \in \mathbb{R}$ and $\lambda, \mu \neq 0$.

Step 3: Substituting (7) in (6) and setting the coefficients of the powers of $\psi$ to be zero, we impose a nonlinear algebraic system in $a_{i}$ and $\lambda$.

Step 4: Solving the system to obtain these values and substituting them into Eq.(7) we receive the exact solutions of
(8).

\section{Applications}

In this section we shall illustrate an example to examine our method.

We consider the following space-time fractional coupled Burgers equations

$$
\begin{aligned}
& D_{t}^{\alpha} u-D_{x}^{2} \alpha_{u+2 u} D_{x}^{\alpha} u+p D_{x}^{\alpha}(u v)=0 \\
& D_{t}^{\alpha}{ }_{v-D_{x}}^{2} \alpha_{v+2 v} D_{x}^{\alpha} \alpha_{v+q} D_{x}^{\alpha}(u v)=0
\end{aligned}
$$

Using the wave transform

$$
u(t, z)=u(\eta), \quad \eta=\lambda t+x,
$$

we receive

$$
\begin{aligned}
& { }_{c}^{\alpha} D_{\eta}^{\alpha} u-D_{\eta}^{2 \alpha} u+2 u D_{\eta}^{\alpha} u+p D_{\eta}^{\alpha}(u v)=0 \\
& { }_{c}^{\alpha} D_{\eta}^{\alpha}{ }^{\alpha-D_{\eta}}{ }^{2 \alpha}{ }_{v+2 v} D_{\eta}^{\alpha} v+q D_{\eta}^{\alpha}(u v)=0
\end{aligned}
$$

By applying the above method yields

$$
\begin{aligned}
& u(\eta)=a_{0}+a_{1} \psi(\eta)+\frac{a_{-1}}{\psi(\eta)}, \\
& v(\eta)=b_{0}+b_{1} \psi(\eta)+\frac{a_{-1}}{b(\eta)}
\end{aligned}
$$

where $\psi$ statisfies the fraction Birnolli equation. Solving the system, we get the following

$$
\begin{aligned}
& a_{0}=-\frac{1}{2} \frac{\lambda(p-1)+c^{\alpha}(p-1)}{p q-1}, a_{1}=\frac{\mu(p-1)}{p q-1}, a_{-1}=0, \\
& b_{0}=-\frac{1}{2} \frac{\lambda(q-1)+c^{\alpha}(q-1)}{p q-1}, b_{1}=\frac{\mu(q-1)}{p q-1}, b_{-1}=0 .
\end{aligned}
$$

Thus we have exact solution of (9) as follows:

$$
\begin{aligned}
& u(\eta)=-\frac{1}{2} \frac{\lambda(p-1)+c^{\alpha}(p-1)}{p q-1}+\frac{\mu(p-1)}{p q-1} E_{\alpha}\left((\mu-\lambda)(\lambda t+x)^{\alpha}\right), \\
& v(\eta)=-\frac{1}{2} \frac{\lambda(q-1)+c^{\alpha}(q-1)}{p q-1}+\frac{\mu(q-1)}{p q-1} E_{\alpha}\left((\mu-\lambda)(\lambda t+x)^{\alpha}\right) .
\end{aligned}
$$

\section{Results and Discussion}

The fractional derivatives in the sense of modified Riemann- Liouville derivative and the fractal index methodare employed for constructing the exact solutions of nonlinear fractional partial differential equations. The existence of the solutions depends on the Banach fixed point theorem. The power of this manageable fractal index method is presented by applying it on two examples. This approach can also be applied to other nonlinear fractional differentional equations.

\section{Conclusion}

The modified subequation method is applied successfully 
for solving the system of nonlinear fractional differentional equations. The performance of this method is reliable and effective and gives more new solutions. This method has more advantages: it is direct and concise. Thus, we deduce that the proposed method can be extended to solve many systems of nonlinear fractional partial diffrentional equations.

\section{References}

[1] I. Podlubny, Fractional Differential Equations, vol. 198 of Mathematics in Science and Engineering, Academic Press, San Diego, Calif, USA, 1999.

[2] A. A. Kilbas, H. M. Srivastava, and J. J. Trujillo, Theory and Applications of Fractional Differential Equations, vol. 204 of North-Holland Mathematics Studies, Elsevier Science B.V., Amsterdam, The Netherlands, 2006.

[3] J. Sabatier, O. P. Agrawal, and J. A. Machado, Advance in Fractional Calculus: Theoretical Developments and Applications in Physics and Engineering, Springer, Dordrecht, The Netherlands, 2007.

[4] V. Lakshmikantham, S. Leela, J. Vasundhara, Theory of Fractional Dynamic Systems. Cambridge Academic Publishers, Cambridge 2009.

[5] D. Baleanu, B. Guvenc and J. A. Tenreiro, New Trends in Nanotechnology and Fractional Calculus Applications, Springer, New York, NY, USA, 2010.

[6] P. R. Gordoa, A. Pickering, Z. N. Zhu, Bucklund transformations for a matrix second Painlev equation, Physics Letters A, 374 (34) (2010) 3422-3424.

[7] R. Molliq, B. Batiha, Approximate analytic solutions of fractional Zakharov-Kuznetsov equations by fractional complex transform, International Journal of Engineering and Technology, 1 (1) (2012) 1-13.

[8] R.W. Ibrahim, Complex transforms for systems of fractional differential equations, Abstract and Applied Analysis Volume 2012, Article ID 814759, 15 pages.

[9] S. Sivasubramanian, M. Darus, R. W. Ibrahim, On the starlikeness of certain class of analytic functions, Mathematical and Computer Modelling, vol. 54, no. -2(2011) pp. 112118.

[10] R. W. Ibrahim, An application of Lauricella hypergeometric functions to the generalized heat equations, Malaya Journal of Matematik, 1(2014) 43-48.

[11] Zheng-Biao Li and Ji-Huan He. "Application of the ractional Complex Transform to Fractional Differential Equations"Nonlinear Science Letters A- Mathematics, Physics and Mechanics 2.3 (2011): 121-126.
[12] Ji-Huan He a, S.K. Elagan and Z.B. Li c, Geometrical explanation of the fractional complex transform and derivative chain rule for fractional calculus Physics Letters A 376 (2012) 257-259.

[13] RabhaW. Ibrahima, and S. K. Elagan, On solutions for classes of fractional differential equations, Malaya J. Mat. 2(4) (2014) 411-418.

[14] J. R. Macdonald, L. R. Evangelista, E. K. Lenzi, and G. Barbero, J. Phys. Chem. C, 115(2011) 7648-7655.

[15] P. A. Santoro, J. L. de Paula, E. K. Lenzi, L. R. Evangelista, J. Chem. Phys. 135(114704)(2011) 1-5.

[16] J.T. Machado, V. Kiryakova, F. Mainardi, Commun. Nonlinear Sci. 16(2011) 1140- 1153.

[17] R. W. Ibrahim, On holomorphic solution for space- and time-fractional telegraph equations in complex domain, Journal of Function Spaces and Applications 2012, Article ID 703681, 10 pages.

[18] R.W. Ibrahim, Numerical solution for complex systems of fractional order, Journal of Applied Mathematics 2012, Article ID 678174, 11 pages.

[19] K. Diethelm, The Analysis of Fractional Differential Equations, Springer-Verlag Berlin Heidelberg, 2010.

[20] S. Zhang, H.Q. Zhang, Fractional sub-equation method and its applications to nonlinear fractional PDEs, Phys. Lett. A, 375 (2011) 1069-1073.

[21] A. N. Kochubei, The Cauchy problem for evolution equations of fractional order, Differential Equations 25 (1989) 967-974.

[22] A. N. Kochubei, Diffusion of fractional order, Differential Equations 26 (1990) 485-492.

[23] R. Metzler, J. Klafter, The random walks guide to anomalous diffusion: A fractional dynamics approach. Phys. Rep. 339 (2000) 1-77.

[24] G. Zaslavsky, Fractional kinetic equation for Hamiltonian chaos. Chaotic advection, tracer dynamics and turbulent dispersion. Phys. D 76 (1994) 110-122.

[25] F. Mainardi, G. Pagnini and R. Gorenflo; Some aspects of fractional diffusion equations of single and distributed order, App. Math. Compu., 187( 1) (2007) 295-305.

[26] Khaled A. Gepreel and Mohamed S. Mohamed, Analytical approximate solution for nonlinear space-time fractional Klein Gordon equation, Chinese physics B, Vol. 22, Issue. 1, 2013, 010201-6.

[27] Hossam A. Ghany and Mohamed S. Mohamed, White noise functional solutions for the wick-type stochastic fractional Kdv-Burgers-Kuramoto Equations, Journal of the Chinese Journal of Physics. Vol. 50, Issue. 4, 2012, pp. 619-627. 